\title{
Cervical Cancer: Cost Management
}

\author{
Marcela Ignacchiti Lacerda \\ Brazilian Army, Rio de Janeiro, Brazil \\ Marcia Menezes da Silva \\ Unimed Rio, Rio de Janeiro, Brazil \\ Angelo Maia Cister \\ FACC/UFRJ, Rio de Janeiro, Brazil
}

\begin{abstract}
The present paper aims to outline the panorama of cervical cancer in Brazil, correlating the particularities of these data and their costs in the scenario of the Brazilian Army, by conducting a retrospective cross-sectional study of the military population attended in the Praia Vermelha Military Polyclinic in Rio de Janeiro, in the period of December 2011 to July 2014. The authors use an auxiliary tool for obtaining the database, the finite population sampling by quotas of age with $95 \%$ confidence interval and sampling error of 5\%. Such a study is relevant when the authors consider the statistical data that cervical cancer is the second most frequent tumor in female population and the fourth cause of death from cancer in Brazil, added to the fact that, if diagnosed early, the chance of cure can reach almost $100 \%$.
\end{abstract}

Keywords: cervical cancer, Papanicolaou, colpocytology, colposcopy, biopsy, cost, Brazilian Army

\section{Introduction}

This article is a literature review of the history and epidemiology of cervical cancer in Brazil and in the world, in addition to a cross-sectional observational study of the profile of such type of cancer in the Brazilian Army scenario, more specifically at the Praia Vermelha Military Polyclinic (PMPV), located in the city of Rio de Janeiro, Brazil. This paper focuses on the quantification, correlation, analysis, and impact of data collected from the perspective of expenses and cost management, and allows the development of general and specific strategies for an improved financial management in this military scenario and its applicability in other media.

The first references to cervical cancer date back to the 15th century BC (before Christ) in Greek and Hindu texts. However, detailed medical texts on the disease and its treatment were only described in the 19th century. In 1900, Cullen introduced the concept of pre-cervical cancer lesions into scientific media. In Germany, in 1924, Hans Hinselmann discovered a device called colposcope. Such device is used in an examination called colposcopy, which appeared as a methodological tool for tracking cervical cancer. Of Greek etymology, colposcopy means kolpos (vagina and Skopeo) to look carefully. With the observation and study of lesions, this method has been improved and goals were established, such as: mapping of cervical lesions, guiding biopsies,

Marcela Ignacchiti Lacerda, M.D., gynecologists, Brazilian Army, Rio de Janeiro, Brazil.

Marcia Menezes da Silva, M.D., gynecologists Unimed Rio, Rio de Janeiro, Brazil.

Angelo Maia Cister, D.Sc., data mining, Federal University of Rio de Janeiro, Rio de Janeiro, Brazil.

Correspondence concerning this article should be addressed to Angelo Maia Cister, Av. Pasteur, 250 room 242-22290-240, Praia Vermelha, Urca Rio de janeiro, RJ, Brazil. 
and guiding the treatment are effective in terms of excision (removal) of the lesions and they are on the cervical tissue, especially in groups of patients who wish to conceive or who have not had children. Thus, in 1927, the first description of pre-cancerous lesions occurred. In 1928, in the United States (US), a Greek naturalized American, Geórgios Papanicolaou (1883-1963) reported that cancer could be diagnosed by smear with vaginal cells collection. However, his work was only recognized in 1943 in the publication of a book discussing the preparation of vaginal and cervical smear, along with Herbert Traut. From such examination, other changes were discovered, and in 1932 Broders noted pre-cancerous lesions around areas with invasive carcinoma, and thus, the term carcinoma was defined in situ.

According to De Carvalho (2010, p. 4), in the face of the discoveries and applications of the two methods mentioned above for detecting cervical cancer, after the 1970s, there was a consequent decrease in cervical lesions, as well as a decrease of mortality rates by 1/3 for cervical cancer compared to the occurrences in 1950 .

Other important events in the history of cervical cancer occurred after 1970: Harald Zur Hausen, Nobel Prize winner for medicine in 2008, proved the etiology of the disease caused by a high-risk sexually transmitted oncogenic (cancer-causing) agent called Human Papilloma Virus (HPV).

Some time later, a molecular biology test called Hybrid Capture was discovered, which enabled the demonstration of viral DNA of oncogenic virus groups (about 14 types) involved in the pathogenesis of cervical cancer, highlighting subtypes 16 and 18, known today to be accountable for approximately 70\% of cervical cancers (INCA, 2013). The addition of the Hybrid Capture of HPV to the Papanicolaou test (Pap smear test) increased the sensitivity and the negative predictive value of the cytological examinations, and the study and isolation of genes, in particular the L1 of HPV, resulted in the production of virus-like particles (VLP), which became an antigenic component of the HPV vaccine. This event allowed the secondary prevention in the form of the Pap smear test to be associated with primary prevention through HPV vaccine. However, more than half of global indices maintain high rates of cervical cancer.

In the 1990s, $70 \%$ of cases diagnosed in Brazil were of invasive (advanced) disease. With the improvement and application of screening methods (collection of cervical-vaginal material = Pap smear test) and diagnosis (colposcopy and biopsy), there was a change in this panorama, and currently, $44 \%$ of cases are diagnosed in the initial lesion or pre-cancerous phase, called in situ (localized). Importantly, women with early diagnosis and treatment can reach nearly a $100 \%$ chance of cure. This fact reinforces the need for application of the examinations already mentioned.

According to the José Alencar da Silva National Cancer Institute (INCA), this year there will be 15,590 new cases, and data show 5,160 deaths from cervical cancer (2013-system info death Brazil).

Based on the above, this kind of tumor is noteworthy, since it is the second most common tumor in the female population, second only to breast cancer, and the fourth leading cause of death by cancer in Brazil, which is equivalent to 4,800 deaths and over 18,000 new cases per year (INCA, 2013).

\section{Etiology, Major Risk Factors for Cervical Cancer, Clinical Manifestations}

Cervical cancer is a tumor developed from alterations in cervical cells. These alterations are called pre-cancerous lesions, if left untreated, they can develop into cancer. There are several risk factors, such as: persisting infection by HPV ontogenetic subtypes, at least 13 HPV subtypes of about 100, which have already been isolated, are involved in the etiopathogenesis of cervical cancer, specially types 16 and 18, which are present in about $70 \%$ of cancer cases. It is estimated that approximately 291 million women worldwide have 
HPV, 32\% of them are infected by oncogenic types, however, not all develop the disease suggesting HPV to be a necessary factor, although not sufficient (INCA, 2013). Other factors described are linked to immunity, genetics, sexual behavior (early sexual debut, high number of partners, and pregnancies), low socio-economic level, smoking, and using medications, such as contraceptive pills and age (women aged over 30 present higher frequency of persistent lesions when compared with those aged below 30). Nevertheless, there are still uncertain mechanisms to determine persistence or regression of cervical lesions. The development of the disease is usually slow and may be asymptomatic in the early stage, and it develops into intermittent vaginal bleeding or bleeding after intercourse, abnormal vaginal secretion, as well as abdominal pain and changes in the urinary and intestinal tract may occur in the later stages.

\section{Prevention, Screening and Diagnosis}

Primary prevention: Aims to avoid or remove risk or causal factors before the pathological mechanism develops into the disease. It impacts the incidence of the disease. In this case there is adoption of barrier methods—-male and female condoms and immunization (HPV vaccine).

Secondary prevention: Early detection of diseases to prevent or reduce the consequences caused by the condition, aiming at the cure, mostly. There are screening tests for cervical cancer: Oncotic Colpocytology or Papanicolaou Test. Diagnostics examination, such as colposcopy with biopsy and examination for the detection of HPV: Hybrid capture.

\section{Methodology}

All medical records of patients treated at the PMPV Gynecology Clinic from December 2011 to July 2014 were analyzed and reviewed, totaling 8,491 (100\%) patients. From such amount, only the medical records of patients referred from general outpatient clinics (Gynecology) to the specialized clinic (Cervical Pathology) were separated, comprising a total amount of 1,868 (22\%) patients (Table 1).

These 1,868 (22\%) patients were stratified as to:

(1) Indication for Performing Colposcopy/Biopsy (diagnostic test): Altered Pap Smear Test (screening test) has 1,365 patients, and 503 patients for other causes (Table 2);

(2) Categories for FUSEX users: Active Military (149), Military Dependents (1,606), Pensioners (39), other (74) (Table 4);

(3) Age group at the time of realization of colposcopy: 15-20, 21-30, 31-40, 41-50, 51-60, 61-70, 71-80 (Table 5).

The database was collected by a "blind" examiner, non-participant of the study (data collector only) using only the number of records without nominal identification of patients in this study, was typed and tabulated on excel for researchers' further interpretation and analysis. As an additional tool to represent the population sample, the authors used the chi-square test of finite population with a $95 \%$ confidence interval and a $5 \%$ sample error.

This work was approved by the medical department, technical, and administrative direction of PMPV in 2013. Also by the direction of FACC/UFRJ to complete the MBA studies in Hospital Management, in February 2015.

\section{Considerations and Results}

The Brazilian Army (PMPV) uses the Brazilian guidelines for the screening and diagnosis of cervical cancer. 
The most widely adopted strategy for screening is the Pap smear test. It is recommended that from the Consensus Meeting held in 1988 by the Ministry of Health, the test should be prioritized and held once a year for women aged between 25 and 60 who have had sexual activity, and after consecutive annual negatives for two years, it should be held every three years (INCA, 2013). This recommendation was based on a study conducted in 1986 by the International Agency for Research on Cancer (IARC). These recommendations do not apply to immunosuppressed women and those with a history of pre-cancerous lesions. However, this screening should be organized in order to obtain a good cost-benefit with high population coverage.

...Countries with coverage over $50 \%$ of the Pap smear done every three to five years have rates of less than three deaths per 100 thousand women a year and for those with coverage over $70 \%$, this rate is equal to or less than two deaths per 100 thousand women per year. (Marchetta \& Descamps, 2004; Baliga \& Shakuntala, 2011)

In the scope of the Praia Vermelha Military Polyclinic, the authors used opportunistic screening performed during medical service. The collection of the Pap smear and other tests, as needed, is performed during gynecological care. Hospital medical assistance for military families is funded by the Brazilian Army health fund (FUSEX). The contribution is required by law under Article 15 of Provisional Measure 2215-10 of August 31, 2001 (15). This fund deducts a percentage, provided for in a specific ordinance (15), from the compensation of the military officers and pensioners of the army; it includes medical and dental treatment, coverage abroad, aeromedical transport, and among others, there is no grace period. FUSEX is a priority of the Army command, which seeks constant improvements in obtaining funds from the federal government, and in the application of the funds available and raised.

When the authors perform the screening test (Pap test), it has an average cost of BRL 14.67 for the Army Health Fund (FUSEX) and the user has a 20\% discount of such value on payroll, i.e. the user "pays" BRL 2.934. From the perspective of the diagnostic examination (colposcopy/biopsy), it has an average cost of respectively BRL 46.94 and BRL 90.40 for FUSEX; BRL 9.39 and BRL 18.08 for the user (Table 6). This examination is performed in cases of altered or suspected Pap smear, symptomatic patients, risk patients, immunosuppressed patients, and among others. Colposcopy must be performed, and according to such findings, it can be supplemented with histopathological examination through cervix biopsy. It is important to say that the costs are until 2014.

Such data and detailed results are in the following tables (from Table 1 to Table 9):

Table 1

Patients Treated at the PMPV Gynecology Clinic From December 2011 to July 2014

\begin{tabular}{lll}
\hline & Absolute values & Percentage \\
\hline Total of cervical cytological examinations performed & 8,491 & $100 \%$ \\
Total of colposcopies performed & 1,868 & $22 \%$ \\
\hline
\end{tabular}

Table 2

Indications X Colposcopies Performed

\begin{tabular}{lll}
\hline Indication & Colposcopy performed & Confirmation HPV cell alterations \\
\hline Altered oncotic colpocytology & 1,365 & 1,248 \\
Altered gynecological examination/history and/or & 503 & 189 \\
presence of HPV lesions = other indications & 1,868 & 1,437 \\
Total &
\end{tabular}


Table 3

Results of Colposcopies

\begin{tabular}{ll}
\hline Colposcopies performed & Results \\
\hline 253 & Normal \\
842 & Low grade lesion (CIN 1) \\
516 & High grade lesion (CIN 2/3) and cancer in situ \\
79 & Invasive cancer \\
178 & No result \\
Total & Altered examinations $=1,437$ \\
\hline
\end{tabular}

Table 4

Patients Who Underwent Stratified Colposcopy by Category

Patients who underwent colposcopy from December 2011 to July 2014

\begin{tabular}{llll}
\hline Active military & Dependents of military & Pensioners & Other \\
149 & 1,606 & 39 & 74
\end{tabular}

Table 5

Patients Undergoing Stratified Colposcopy by Age Group

\begin{tabular}{lllllll}
\hline Colposcopy & \multicolumn{1}{l}{} \\
\hline $15-20$ years & $21-30$ years & $31-40$ years & $41-50$ years & $51-60$ years & $61-70$ years & $71-80$ years \\
23 & 213 & 1105 & 486 & 32 & 7 & 2 \\
\hline
\end{tabular}

Table 6

Prices in BRL of Examinations for PMPV (FUSEX/Users) in 2014

\begin{tabular}{llll}
\hline & $\begin{array}{l}\text { Oncotic } \\
\text { colpocytology }\end{array}$ & Colposcopy & $\begin{array}{l}\text { Colposcopy }+ \\
\text { biopsy }\end{array}$ \\
\hline FUSEX & 11.74 & 37.55 & 72.32 \\
User (20\%) & 2.93 & 9.39 & 18.08 \\
Total & 14.67 & 46.94 & 90.40 \\
\hline
\end{tabular}

Table 7

Absolute Values/Costs During the Period of Study

\begin{tabular}{llll}
\hline & Oncotic colpocytology & Colposcopy + biopsy & $\begin{array}{l}\text { Colposcopy }+ \\
\text { biopsy + oncotic } \\
\text { colpocytology }\end{array}$ \\
\hline FUSEX & $99,650.38$ & $135,093.76$ & $234,744.14$ \\
User (20\%) & $24,912.60$ & $33,773.44$ & $58,686.04$ \\
Total & $124,562.98$ & $168,867.2$ & $293,430.18$ \\
\hline
\end{tabular}

Table 8

Patients Stratified by Age Group Recommended to Conduct Screening Test

\begin{tabular}{lll}
\hline Age group (years) & Oncotic colpocytology (annual) & Oncotic colpocytology (>2 years) \\
\hline $21-30$ & $57.9 \%$ & $42.1 \%$ \\
$31-40$ & $63.63 \%$ & $36.37 \%$ \\
$41-50$ & $37.93 \%$ & $62.17 \%$ \\
$51-60$ & $28.57 \%$ & $71.43 \%$ \\
\hline
\end{tabular}


Table 9

Outcomes According to the Age Group for Patients Who Underwent Screening Examination for a Period Longer Than Two Years

\begin{tabular}{lll}
\hline Oncotic colpocytology (> 2 years) & & \\
\hline Age group (years) & HFS/conization & Hysterectomy (TAH) \\
$21-30$ & $25 \%$ & $0 \%$ \\
$31-40$ & $63.7 \%$ & $4.17 \%$ \\
$41-50$ & $40.7 \%$ & $59 \%$ \\
$51-60$ & $0 \%$ & $99 \%$ \\
\hline
\end{tabular}

\section{Discussion}

The authors extracted samples of 1,868 patients (22\%) from the total female population served in the gynecology outpatient clinic during the study period, who underwent examination for diagnosis (colposcopy) of cervical cancer, such samples comprise:

- 1,365 patients $(73 \%)$ who were indicated since their screening test (Pap smear) was altered;

- among them, 1,248 (91.4\%) confirmed cellular changes consistent with the presence of HPV. These data reaffirm the Pap test/Pap smear as an effective test for population screening for cervical cancer (Tables 1, 2, and 3).

Patient profile:

- As for the category within the FUSEX: 1,606 (86\%) are military dependents. This profile can be explained by the history of the Brazilian Army, which up to present year (2014), the Agulhas Negras Military Academy (AMAN), which trains official career combatants, only admits men in their personnel. Therefore most served women are married to military officers and/or are military officers' daughters (86\%) — dependents, or widows and/or daughters of deceased military officers (4\%)-pensioners. The percentage of active female military officers (8\%) consists of officers and sergeants from the health department and complementary departments (administration, library science, accounting, media, law, nursing, computer science, psychology, veterinary medicine, and teaching) and their dependents, respectively graduated from the Army's Health School (Essex) and the Army School for Complementary Training (EsFCEx). However, not only do these schools train men and women, they annually train fewer women than AMAN trains men.

- As for the age group: 1,605 (85.9\%) are aged 31 to 60. Patients aged under 20 and over 60 represent about $5 \%$ of performed colposcopies; such number is not significant for this study, as well as other population studies, which reinforces the age group 25 to 60 recommended by the Brazilian Ministry of Health for carrying out the examination (Tables 4 and 5).

- On the financial scope, the authors realize that when comparing the costs for both FUSEX as for its users, it can observe that, on average, the screening test costs three times less than the isolated diagnostic examination (colposcopy) and that when associating examinations is required (colposcopy + biopsy) this value is up to six times lower. Therefore, if screening tests are performed at certain times and recommended for this condition, there may be a decrease in spending on diagnostic examinations (Table 7).

Such fact is proven by Tables 8 and 9 where we observed that most patients ranging in age from 41-50 and 51-60 did not take annual screening tests and had more severe outcomes. That is, at the time of their diagnostics, they already presented advanced stage lesions, which could not be approached from the standpoint of treatment with conservative surgery, sometimes performed in outpatient clinics, such as high frequency surgery HFS/conization. Therefore, these patients whenever under therapeutic possibility and benefit for surgical 
approach are submitted to a hysterectomy, which is a medium to large surgery strictly performed in hospital environment. This fact also has great impact on the cost, since among other things, it have a greater number of professionals involved in the procedure, higher spending of material, longer hospital stays, and longer bed occupancy, which leads to less turnover, greater possibility of complications, and the need for additional treatment, such as radiotherapy, for example. Hysterectomy costs about four to five times more than the HFS/conization (AMB 92) 14.

Some peculiarities of the population in question that can act as a bias of confusion in the study are worth noting: when it comes to military population, the establishment of statistical data, the performance of examinations, whether screening and/or diagnosis, may have biases on research and analysis, since the authors have a number of unique challenges to the lifestyle that the military profession imposes. For instance, difficulties in establishing and recruiting a target population, as the authors do not have a fixed population. In this study, 178 patients who underwent colposcopy did not return and have not been found for delivering the result.

The polyclinic, as well as other military health units, serves military population on a demand system, any Army Health Fund (FUSEX) user of the national territory, resident or not in Rio de Janeiro can be served as it occurs in other Army health units throughout Brazil. Many users take up residence for short period of time and move to another city, state, depending on the transfers of their husbands, or their own transfers, thus not taking up fixed residences for a specified time. Such fact is often inherent to the military career. So, many times the authors miss the opportunity of recruiting women who should do screening tests, we should ensure the approach in women with abnormal tests, as well as the quality measurement of procedures performed and follow up of the progress of diseases. Such factors may unnecessarily burden the system, since there are difficulties in the deployment and implementation of effective policies for primary and secondary prevention.

\section{Conclusion}

It can be concluded that prevention is the best way to reduce the statistics of the increase of cervical cancer, through regular Pap tests/Pap smears, although, unfortunately, deaths from cervical cancer are very frequent in Brazil, which denotes late diagnosis and treatment. The authors conclude that if the authors do periodic screening tests in the population of this study, the number of cervical cancer cases, which have been diagnosed belatedly, will decrease, as it will be the expenses in the short, medium, and long term for the Brazilian Army, enabling the use of funds and changes in the budgetary allocation for other related areas. The authors, therefore, propose the employment of active search for patients who do not meet the standards of periodicity of the cervical cancer screening tests, through the implementation of a nationwide computerized tool that allows this search. That fact will decrease the overall costs and the number of women with this type of cancer in the Brazilian Army, in the medium and long term, whereby this experience will cause an impact and take the implementation of electronic medical records to other media.

\section{References}

Marchetta, J., \& Descamps, P. (2004). Colposcopia (1st ed.). Rio de Janeiro: Revinter.

De Carvalho, N. S. (2010). Newton Sergio. Patologia do Trato Genital Inferior e Colposcopia. São Paulo, Belo Horizonte, Rio de Janeiro: Atheneu.

Baliga, B., \& Shakuntala. (2011). Colposcopia principios e práticas (2nd ed.). Rio de Janeiro: Revinter.

INCA. (2013). Diretrizes Brasileiras para o rastreamento do câncer do colo do útero/instituto nacional do câncer. Coordenação geral de ações estratégicas. Divisão de apoio à rede de atenção oncológica. Rio de Janeiro: INCA. 